\title{
Relación entre la cargabilidad en el dominio del tiempo y la cargabilidad espectral en un estudio de IP realizado por una empresa de exploración el sector de Checa Alta, Valle de Tambo Arequipa, 2015
}

\author{
Relation between time-domain chargability and spectral chargability in an IP \\ survey conducted by an exploration company in the high sector Checa, Valley \\ of Tambo Arequipa, 2015
}

Ricardo Fabian Peláez Mc Evoy', Jesús Alberto Torres Guerra²

Recibido: 15/12/2020 - Aprobado: 26/10/2021 - Publicado: 23/12/2021

\begin{abstract}
RESUMEN
Los estudios de geofísica orientados a la caracterización juegan un papel muy importante para proporcionar un mejor entendimiento de la estructura interna de la tierra y específicamente de una nueva zona de estudio y como consecuencia poder mapear sus propiedades, sus características y los efectos que generan los procesos de alteración geológica. Esto finalmente generará en una mejor interpretación geológica - geofísica en la zona nueva zona de estudio. En este trabajo de investigación se está utilizando los datos de cargabilidad registrados en el dominio del tiempo; primeramente, se utiliza los valores registrados a través de las 20 ventanas muestreadas en el tiempo y esta curva de decaimiento obtenida es la que se utiliza para determinar la función de transferencia que expresa el comportamiento del subsuelo al aplicar una corriente y observar su cargabilidad. Seguidamente se utiliza esta función de transferencia y se aplica la transformada de Fourier para descomponer esta nueva señal en sus valores espectrales. Estos valores espectrales extraídos de la función de transferencia contienen valores de amplitud, Fase y frecuencia angular. Con estas nuevas variables se ha generado 3 cubos adicionales y esto ha hecho posible tener más elementos y variables que ayuden en una mejor caracterización e interpretación de la zona de estudio. Lo importante de este trabajo de investigación es la forma como se utiliza los datos registrados en el tiempo y a través de una transformada de Fourier se cambia al dominio espectral que poseen características sensibles para cada tipo de roca 0 suelo. Esto ha permitido poder entender cómo se comporta un medio al aplicarle un campo eléctrico y mediante estos resultados podemos otorgarles un valor agregado y adicional a los datos de cargabilidad registrados en el tiempo. Todo lo señalado anteriormente se ha conseguido utilizando una serie de algoritmos que se han desarrollado en MATLAB para finalmente obtener las variables frecuencia angular, amplitud y fase. Finalmente, estos resultados se observan con mejor claridad con la variable amplitud donde se puede observar una diferenciación entre un tipo de roca masiva, una roca fracturada, zonas de alteración y zonas de baja compactación. Con respecto a la variable fase se puede confirmar una diferenciación de bloques con un buen alineamiento de la falla confirmando el rumbo y azimut de esta.
\end{abstract}

Palabras claves: Cargabilidad en el dominio del tiempo; cargabilidad espectral; transformada de Fourier.

\begin{abstract}
Geophysical studies oriented to characterization play a very important role in providing a better understanding of the internal structure of the earth and specifically of a new study area and consequently being able to map its properties, its characteristics and the effects generated by the processes of geological alteration. This will finally generate a better geological - geophysical interpretation in the new study zone. In this research work the chargeability data registered in the time domain is being used, firstly the values registered through the 20 windows sampled in time are used and this decay curve obtained is the one used to determine the function transfer that expresses the behavior of the subsoil when applying a current and observing its chargeability. This transfer function is then used, and the Fourier transform is applied to decompose this new signal into its spectral values. These spectral values extracted from the transfer function contain values of amplitude, phase, and angular frequency. With these new variables, 3 additional cubes have been generated and this has made it possible to have more elements and variables that help in a better characterization and interpretation of the study area. The important thing about this research work is the way in which the data recorded over time is used and through a Fourier transform it is changed to the spectral domain that have sensitive characteristics for each type of rock or soil. This has allowed us to understand how a medium behaves when an electric field is applied to it and through these results, we can give an added and additional

1 Bachiller e Ingeniero Geofísico de la Universidad Nacional de San Agustín de Arequipa, Perú.

Autor para correspondencia: ricpelaez 6363@hotmail.com - ORCID: https://orcid.org/0000-0002-2724-7375

2 Universidad Nacional Mayor de San Marcos, Facultad de Ingeniería Geológica, Minera, Metalúrgica y Geográfica, Unidad de Posgrado, Lima, Perú. Docente. E-mail: jorresgu@unmsm.edu.pe - ORCID: https://orcid.org/0000-0001-8186-5249
\end{abstract}


value to the chargeability data recorded over time. Everything mentioned above has been achieved using a series of algorithms that have been developed in MATLAB to finally obtain the variables angular frequency, amplitude, and phase. Finally, these results are observed with better clarity with the variable amplitude where a differentiation can be observed between a type of massive rock, a fractured rock, alteration zones and zones of low compaction. Regarding the phase variable, a differentiation of blocks with a good alignment of the fault can be confirmed, confirming the bearing and azimuth of the fault.

Keywords: Time domain chargability; spectral chargability; Fourier transform.

\section{INTRODUCCIÓN}

El método de polarización inducida (Seigel et al., 2007) ha madurado en los últimos 50 años desde una herramienta que busca anomalías relativamente simples hasta utilizarlo como un método analítico cuantitativo. En todos los estudios realizados con el método de polarización inducida el objetivo principal ha sido la detección y búsqueda de metales base como los sulfuros y en otros casos como un método para determinar zonas de arcillas. En esta oportunidad el trabajo de investigación está orientado a demostrar el valor adicional que contiene las curvas de decaimiento obtenidas en campo que reflejan los valores de cargabilidad y mediante un método de transformación del dominio del tiempo al dominio de la frecuencia, generar nuevas variables que permitan caracterizar una nueva zona de estudio. Este tema de investigación nos permite demostrar que las señales registradas en el tiempo contienen información muy importante que ayudan y generan valor agregado a los datos de cargabilidad.

Esta relación es muy importante porque la cargabilidad y su respuesta espectral descompuesta en tres elementos, tales como la frecuencia, amplitud y fase permite ingresar al dominio espectral y esto abre la posibilidad de poder generar varios productos que ayudaran en la interpretación geológica y relacionarlas con algún aspecto estructural, algún cambio textural en las rocas y finalmente encontrar la relación de la anomalía de cargabilidad con algún aspecto de mineralización que es de interés en los estudios de exploración.

Hay una diferencia entre los datos registrados en el dominio espectral aplicando una fuente de corriente alterna donde se genera frecuencias controladas y los datos registrados en el tiempo de manera convencional utilizando una fuente de corriente pulsante, que es nuestro caso. En este segundo caso se está encontrando una respuesta espectral intrínseca y natural que es propia del terreno y que está relacionada a la cargabilidad registrada.

Mientras que las mediciones espectrales de IP generadas con una fuente alterna son aquellas que también se registran en el dominio del tiempo, y son afectadas por frecuencia que corresponden a efectos electromagnéticos (EM) que podemos considerarlos como ruido y es necesario corregirlos y filtrarlos para que no altere los valores primarios, propios de la zona en estudio.

\subsection{Ubicación del estudio de IP}

El área de investigación se encuentra ubicada en el departamento de Arequipa, provincia de Islay. En la figura 1 se muestra la ubicación del estudio y en la figura 2 se muestra las líneas de IP. El tiempo de viaje de la ciudad de Arequipa a la zona de estudio tomo 2 horas y media a

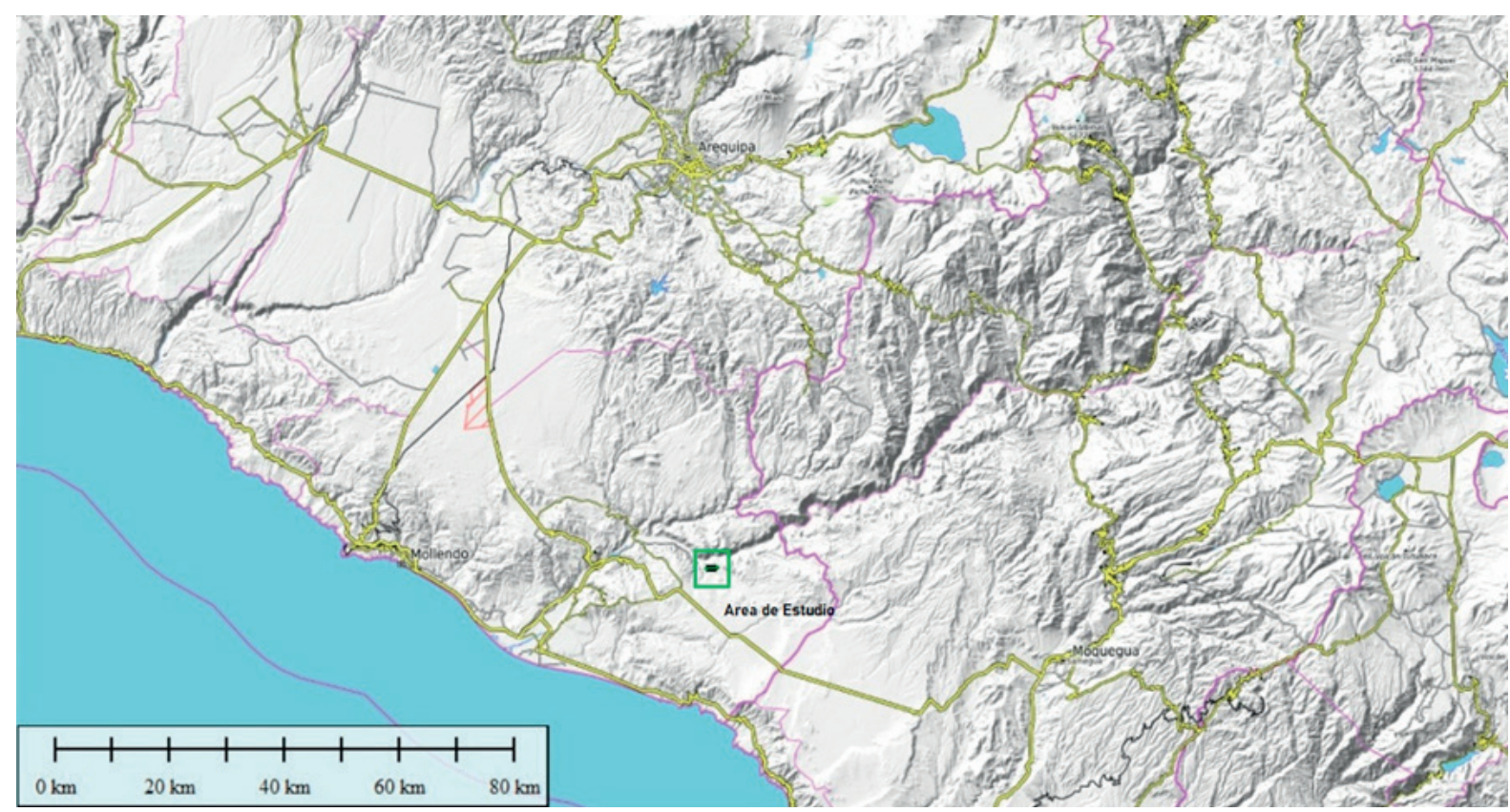

Figura 1. mapa de ubicación de la zona de estudio. 


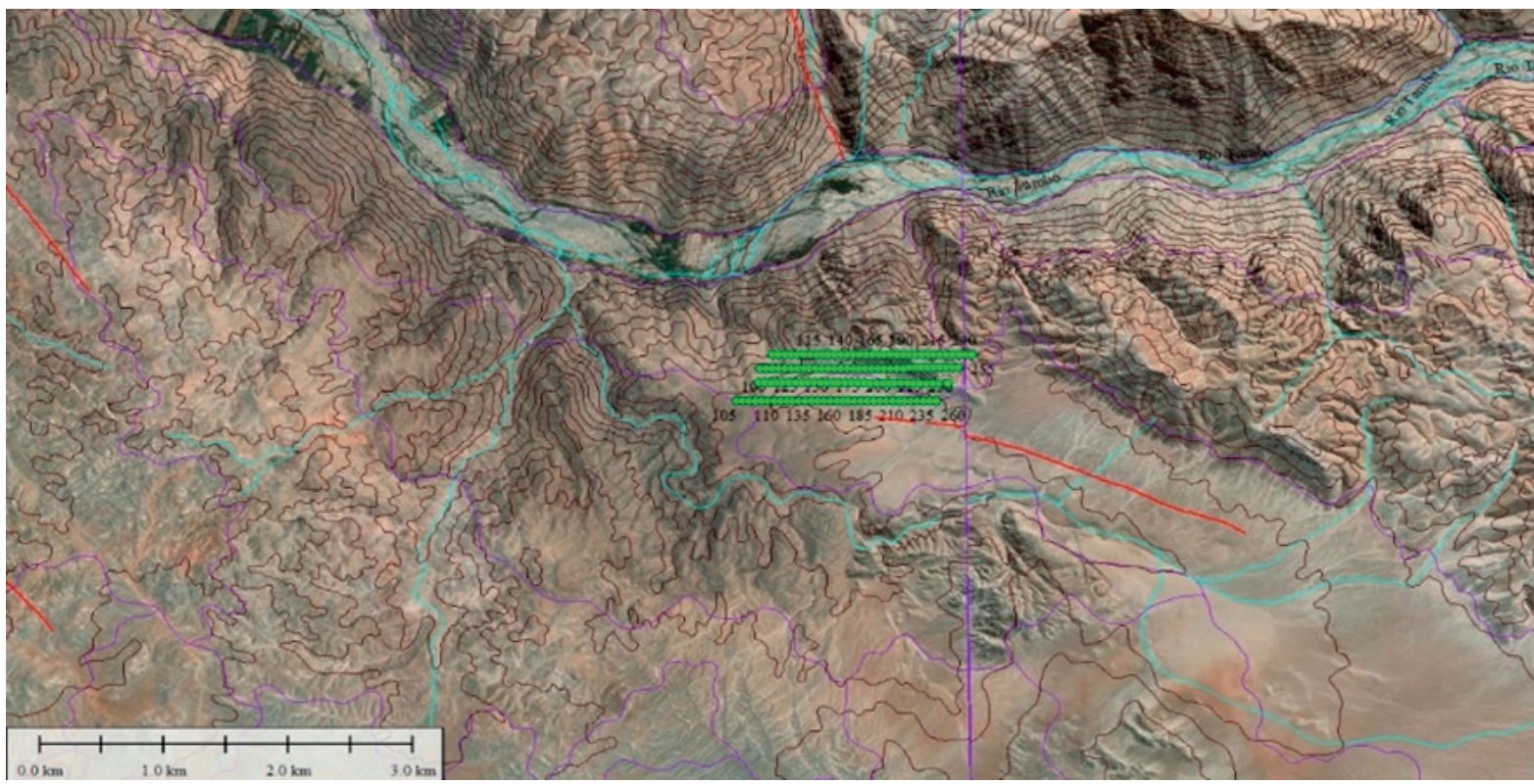

Figura 2. mapa de ubicación de las líneas de IP.

través de la vía de la Panamericana sur con dirección a la ciudad de Moquegua. El centro de operaciones se ubicó en el pueblo de Chucarapi, lugar desde donde se operó diariamente y el tiempo de viaje fue de 1 hora y media a la zona de trabajo. Las coordenadas se expresan en el sistema UTM, Datum WGS 84 del hemisferio Sur, los vértices del área de estudio son las siguientes:

$$
\begin{aligned}
& 231944.7,8114521.6 \\
& 234057.4,8114507.4 \\
& 234064.4,8113798.5 \\
& 231944.7,8113805.5
\end{aligned}
$$

\subsection{Contexto geológico de la zona de estudio}

El complejo basal de la costa (Bellido Bravo \& Narvaez, 1960) ubicado en la costa sur del Perú, entre los poblados de San Juan de Marcona, Mollendo e Ilo forma la Cordillera de la Costa. Este tipo de rocas también son conocidas como macizo de Arequipa (Cobbing \& Pitcher, 1972). El complejo basal de la costa está constituido por rocas del Meso-proterozoico y Paleozoico, tales como gneis, esquistos, dioritas, granitos y migmatitas. Este basamento rocoso ha sufrido una deformación continua y una cristalización del granito por lo que se considera como un ortogneis y ha sufrido inyecciones de granitos rojos que corresponden a una edad entre los 2000 a 600 Ma. (Cobbing E. , 1985).

INGEMMET ha realizado dataciones radiométricas en muestras de zircones determinando edades de 1862.6 Millones de años (+-8.1) para un gneis diorítico y de 996.4 Millones de años (+-5.3) para rocas graníticas, asignándoles una edad que corresponde al Mesoproterozoico al complejo basal de la costa. Estas rocas están relacionadas a depósitos de feldespatos en forma de diques.
Seguidamente encontramos al Grupo Cabanillas de manera discordante sobre el gneis Mesoproterozoico, este grupo está constituido por una secuencia de areniscas cuarzosas que están intercaladas con arcillitas silicificadas del Devónico. Inmediatamente tenemos a la Formación Chocolate del Jurásico inferior constituida por lavas andesíticas y dacíticas con algunos horizontes de conglomerados, sus afloramientos se ubican en Punta de Bombón y Clemesí.

Dentro de los volcánicos eruptivos, el volcánico Chocolate se considera uno de ellos.

La Formación Guaneros del Jurásico superior está en discordancia al Volcánico Chocolate y está compuesta por lavas andesíticas y dacíticas porfiríticas, aglomerados y areniscas, su origen eruptivo está ubicada más al Oeste en una zona ahora cubierta por el océano Pacifico.

Un nuevo ciclo volcánico se generó en un periodo entre el Cretácico superior e inicios del Terciario y esto originó los extensos depósitos del Grupo Toquepala afloran desde el límite del rio Tambo hacia el Sureste, conformando las zonas bajas y medias del lado occidental andino. Este depósito muestra varias discordancias entre sus varias fases, que indican interrupciones en sus ciclos, seguidos por varios procesos erosivos, que están constituidos por brechas de lava y flujos piroclásticos que muestran una composición andesítica y dacíticas.

En la Formación Moquegua se observa la sedimentación que se inició en el Oligoceno temprano, encima de un terreno irregular establecido por sus rocas volcánicas, la Formación Camaná formada de areniscas y arcillitas de color crema y abigarradas, del Oligoceno medio, la Formación Millo constituida por conglomerados polimícticos con un origen diverso de las gravas redondeadas, areniscas conglomeráticas, tobas y cenizas volcánica, las cuales recubren una importante área de 
estudio y por finalmente viene la disposición de depósitos cuaternarios que se encuentran en las partes altas.

Durante el Mesozoico inferior se intruyó la súper unidad Punta Coles constituida principalmente por dioritas y granodioritas de 190 Ma. (Pitcher \& Bussell, 1985); está súper unidad es el componente más antiguo del Batolito de la Costa, la cual intruyó a la Formación Chocolate (Jenks, 1948), en el Cretáceo tardío y el Terciario medio se emplazó la super unidad Ilo compuesta de diorita, diorita cuarcífera, granodiorita hornblendica de $103 \mathrm{Ma}$. (Pitcher \& Bussell, 1985), la cual intruyó a los intrusivos de la súper unidad Punta Coles y en el Cretáceo Superior se emplazó la súper unidad Yarabamba compuesta por intrusivos de composición diorítica, monzonítica y granodiorítica con hornblenda.

\subsection{Objetivos de la investigación}

- Determinar la relación entre la cargabilidad en el dominio del tiempo y su respuesta espectral en un estudio de Polarización Inducida realizado en el sector de Checa Alta, Valle de Tambo, Arequipa.

- Determinar la relación que existe entre la cargabilidad y la respuesta espectral de la frecuencia, la fase y la amplitud en un estudio de polarización inducida.

\section{MÉTODOS}

El método aplicado para obtener los resultados en este estudio consistió en utilizar la transformada de Fourier que es una herramienta de gran valor para analizar las funciones no periódicas. Por lo tanto, la curva de decaimiento no considerada como una función no periódica y que ha sido registrada en el dominio del tiempo se transformó al dominio de la frecuencia utilizando la transformada de Fourier mediante una función de transferencia obtenida de los datos registrados.

Esta transformada producirá valores de amplitud, frecuencia y valores de ángulo de fase. Intuitivamente, uno espera una relación uno a uno entre la cargabilidad en el dominio del tiempo y el ángulo de fase en el dominio de la frecuencia, pero a pesar del hecho de que esta relación es reconocida hay pocos estudios realizados al respecto. Se observa diferencias en la respuesta de la polarización inducida espectral para diferentes tipos de rocas, y particularmente para casos de rocas mineralizadas que exhiben una dispersión en la frecuencia de manera significativa (Sogade et al., 2006).

Este estudio de investigación tiene un carácter descriptivo ya que permite obtener las características y propiedades del fenómeno de la Cargabilidad observada. El tipo de diseño que corresponde a este estudio es no experimental, por lo tanto, no se manipula la variable Cargabilidad, solo se registra esta variable de manera indirecta como una respuesta del terreno después de haber inyectado una corriente para generar un campo eléctrico y después registrar la diferencia de potencial. En otras palabras, se interpreta la realidad de una situación ya existente no provocada sino como una respuesta del terreno en estudio. Este diseño no experimental, se clasifica en una modalidad transversal que tienen lugar cuando se recolectan los datos en un solo momento. El presente estudio se encuadra en esta investigación transversal, dado que se obtienen las variables espectrales y se analiza su relación después de aplicar una transformación matemática utilizando una función de transferencia como respuesta del terreno.

La metodología aplicada en el presente trabajo de investigación estuvo compuesta por las siguientes fases:

- Concepción de la idea,

- Descripción del problema,

- Marco teórico,

- Alcance de la investigación,

- Definición y formulación de las hipótesis,

- Diseño de la investigación,

- Selección de muestras,

- Recolección de datos,

- Análisis de los datos y

- Resultados de la investigación.

\subsection{Polarización inducida}

El método de polarización inducida consiste primero en inyectar corriente y generar una polarización a diferentes niveles en profundidad en el subsuelo. Hay medios en la cual debido a la presencia de minerales tales como los sulfuros generan una cargabilidad. Seguidamente se hace mediciones del decaimiento de este sobre voltaje en el tiempo después de la cesación del pulso de corriente (Método del dominio del tiempo) (Sheriff, 2002).

Hay dos mecanismos por los cuales el comportamiento natural del medio tiene un comportamiento tipo condensador. El primero se denomina polarización de membrana o también conocido como electrolítico y el segundo como polarización por grano o de electrodos. Este último puede estar asociado con minerales potencialmente económicos, mientras que el primero no lo es.

\subsection{Polarización de membrana}

La polarización de membrana se genera por la impedancia de la interfase entre dos medios y es el resultado para ciertas regiones que poseen conducción normal en una trayectoria de poro, una capa de difusión es muy gruesa para bloquear dicho poro, de manera que se realiza una transferencia de iones de cierto tamaño y una polaridad, reduciendo el movimiento de las cargas generando un almacenamiento que crea una "membrana" polarizada. En general este tipo de polarización ocurre en suelos que contienen arcillas, debido a que poseen una capacidad de intercambio iónico alto que bloquea parcialmente la trayectoria de la solución. Por lo tanto, la polarización de membrana es un efecto de la polarización inducida que se observa comúnmente en estudios de mapeos de contaminación (Sogade, 2006). 


\subsection{Polarización de granos}

Cuando los iones fluyen a través del fluido de los poros en respuesta a un potencial aplicado, ellos pueden encontrar granos electrónicamente conductores, por ejemplo, los sulfuros. Además, ellos pueden formar una barrera con la cual los iones pueden interactuar eléctricamente. Para que la corriente fluya, los iones en un lado de la barrera deben intercambiar electrones con el conductor grano, que a su vez intercambia electrones con iones en el otro lado de la barrera. El circuito eléctrico localmente consiste en una combinación de conducción iónica y electrónica e implica una reacción química entre el mineral y la solución (el fluido en los poros) (Dentith \& Mudge, 2015).

\subsection{Cargabilidad}

Este valor de cargabilidad se observa cuando una corriente estacionaria se inyecta en el subsuelo mediante dos electrodos y luego esta corriente se interrumpe. Esta tensión no retornará a cero en forma instantánea, sino que decaerá lentamente en el tiempo, lo que indica que esta carga ha sido almacenada en el medio donde se inyecto esta corriente.

Esta Cargabilidad Integral se define como el área bajo la curva de descarga donde V (t) y Vm tienen las mismas unidades y la Cargabilidad es expresada en milisegundos (Telford \& Geldart, L., \& Sheriff, 1931).

$$
M=\frac{1}{V m} \int_{t 1}^{t 2} V(t) d t
$$

\subsection{Equipos utilizados}

Instrumentación GDD INC

- $\quad$ Receptor digital GDD de 32 canales.

- $\quad$ Modelo GRx8-32

- Ventanas programables

- Modos disponible utilizado Cole-Cole
- Potencia del transmisor 5000W (Walcer Geophysics Ltda.)

- Corriente máxima: 10Amp

- Dominio de Tiempo: 1, 2, 4 y 8 segundos.

- $\quad$ Frecuencias de salida 0.125 Hertz

- Voltajes de salida: 200, 300, 500, 700, 1000, $1500,2000,2500$ y 3000 Voltios

Este trabajo de investigación se ha divido en 4 partes.

- Determinación de la Función de transferencia

- Aplicación de la Transformada de Fourier a los datos

- Cálculo de las variables de Frecuencia-Amplitud y Frecuencia-Fase

- Establecer la relación entre los valores de la cargabilidad y la cargabilidad espectral.

\subsection{Determinación del modelo exponencial}

La determinación del modelo exponencial es el punto de partida y tomando en consideración los datos registrados en las 20 ventanas en el dominio del tiempo se transforma estos valores como logaritmos en base 10. Seguidamente se utiliza la metodología por mínimos cuadrados para obtener los coeficientes $a_{0}$ y $a_{1}$ y determinar la función exponencial que expresa el comportamiento de la curva de decaimiento en cada punto a lo largo de cada perfil.

Una vez obtenidos los valores de los coeficientes $a_{1} \mathrm{y}$ aplicando el inverso del logaritmo en base 10 al valor de $a_{2}$, se obtendrá los dos coeficientes que representan la función exponencial final que es la que representa la siguiente curva de decaimiento. (Figura 3).

$$
\begin{aligned}
Y= & 89.183 X^{-0.629} \\
& R^{2}=0.9845(3)
\end{aligned}
$$

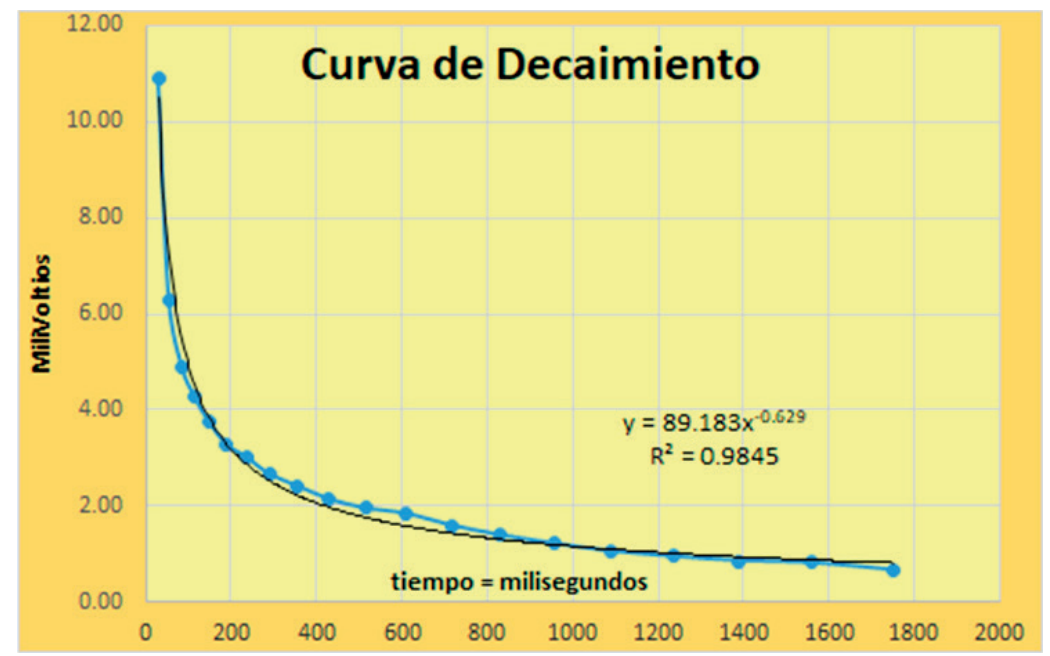

Figura 3. Curva de decaimiento 
La primera ecuación corresponde a la ecuación de potencias, mientras que la segunda corresponde al coeficiente de correlación o de Pearson que presenta un valor de 0.9845 , es decir es próximo a 1. Este valor expresa la relación entre las dos variables. Este valor vario entre 0 y 1 , mientras este valor esté más cerca de 1 habrá una mayor similitud entre las dos ecuaciones. En la mayoría de las comparaciones de los datos de las curvas este valor ha sido muy próximo a 1.

\subsection{Regresión polinomial}

La Regresión Polinomial se considera un caso especial de la Regresión Lineal, en la cual se extiende el modelo lineal al agregar predictores adicionales que se obtienen cuando se elevan cada uno de los predictores originales a una potencia. Este método extiende la Regresión Lineal a una relación que no es lineal entre las variables dependientes e independientes reemplazando un modelo lineal por una función polinomial. Por lo tanto, el método de regresión polinomial es el más adecuado para ajustar un tipo de datos que exhiben un comportamiento curvilíneo.

Esto permite de una forma directa realizar el modelamiento de las curvas sin tener que proponer modelos complicados no lineales. Se realizan los ajustes lineales dentro de un espacio de mayor dimensión construido con estas funciones básicas, este modelo tiene la facilidad de adaptarse a una serie de opciones de datos de manera más amplia.

En nuestro caso se planteó obtener un polinomio de segundo grado o también llamado cuadrático.

$$
y=a_{0}+a_{1} x_{i}+a_{2} x_{i}^{2}+e
$$

\subsection{Determinación de la función de transferencia}

La función de transferencia es una función matemática lineal o no lineal que utiliza los modelos matemáticos para caracterizar la zona de estudio y permite representar el comportamiento dinámico y estacionario de cualquier sistema.

Una vez que se han determinados los coeficientes del polinomio cuadrático a través de la regresión polinomial para cada punto del perfil se pudo definir esta función como la función de transferencia y a esta función se aplicará la transformada de Fourier.

$$
y=a_{0}+a_{1} x_{i}+a_{2} x_{i}^{2}
$$

Al aplicar la transformada de Fourier a la función de transferencia, los nuevos datos extraídos de este proceso generaran las nuevas variables de frecuencia vs Amplitud y frecuencia vs Fase.

\subsection{Relación de la cargabilidad con la ecuación exponencial}

Adicionalmente a los resultados obtenidos anteriormente, se ha propuesto la siguiente ecuación integral, en la cual se iguala dos funciones obtenidas. La primera es el valor de la cargabilidad $\mathrm{M}$ obtenida por integración de la curva de decaimiento y la segunda ecuación corresponde a la ecuación exponencial obtenida expresada en la variable tiempo. Por lo tanto, podemos proponer lo siguiente:

$$
\frac{\partial M}{\partial t}=b X^{a}
$$

Expresando el valor de $\mathrm{x}$ en valor del tiempo

$$
\frac{\partial M}{\partial t}=b t^{a}
$$

Aplicando integración en ambos lados de la ecuación

$$
M=b \int_{t 1}^{t 2} t^{a} d t
$$

Esta ecuación expresa la relación entre la cargabilidad y la ecuación exponencial obtenida mediante el procesamiento de los datos registrados en campo que determina los valores de cargabilidad. Esta nueva relación es la que define la nueva curva de decaimiento expresada en tiempo y a través de ella podemos obtener los tiempos correspondientes a cada punto en profundidad para nuestra caracterización de la zona de estudio.

\subsection{Transformada continua de Fourier}

Una transformada de Fourier es una operación que convierte funciones en el dominio del tiempo al domino de la frecuencia. Esta es la fórmula que se ha utilizado y define el principio de esta transformada (Quinquis, 2013).

$$
x(f)=\int_{-\infty}^{\infty} x(t) e^{-j 2 \pi f t} d t
$$

\section{t : Tiempo}

\section{$\mathrm{f}=$ Frecuencia en Hertz}

\section{$x(t)$ : Funcion de Transferencia}

$$
\mathrm{e}^{-\mathrm{j} 2 \mathrm{ft}} \mathrm{dt} \text { : Funcion Kernel }
$$

\section{$X(f)$ : Espectro en funcion de la frecuencia}

Una transformada de Fourier inversa (IFT) se convierte del dominio de la frecuencia al dominio del tiempo. Ambas son consistentes (Quinquis, 2013).

$$
(t)=\int_{-\infty}^{\infty} \mathcal{X}(\mathbf{f}) \mathrm{e}^{-\mathrm{j} 2 \pi \mathrm{ft}} \mathrm{df}
$$

\section{RESULTADOS}

Los estudios de Polarización Inducida se muestran sobre la variable de cargabilidad. En la mayoría de los resultados obtenidos se obtiene una relación directa entre la concentración de sulfuros y los valores de cargabilidad. El tipo y grado de mineralización no es fijo y se han propuesto algunas tablas para mostrar estas relaciones de porcentaje (Telford \& Geldart, L., \& Sheriff, 1931).

Los valores de cargabilidad encima de 20 milisegundos muestran valores de $0.5 \%$ y valores mayores a 30 milisegundos valores de $1 \%$ en sulfuros totales. No se ha definido una relación directa entre los valores obtenidos 
de magnetita y los valores de la cargabilidad. Esto se debe a que la magnetita es un mineral de origen magmático formado por diferenciación magmática o metamorfismo de contacto. Mientras que los sulfuros como la pirita y la calcopirita son de origen hidrotermal y metamórfico. Se puede encontrar una asociación entre ambos, pero no es una relación directa (Aguilef Carmona, 2012).

Se realizó un control de calidad a los datos de cargabilidad registrados en campo porque se detectó que había un acople de ruido de carácter telúrico que enmascaraba a las curvas de decaimiento. Por lo tanto, se generó un algoritmo (Filtro de baja frecuencia) para eliminar este ruido y limpiar la curva de decaimiento, este proceso se aplicó antes de la inversión de los datos.

En el procesamiento de datos e inversión se generó un cubo 3D con los datos de cargabilidad y resistividad. El objetivo prioritario fue identificar de acuerdo con la anomalía de cargabilidad algún depósito de sulfuros, asociado con una zona de alteración, este reconocimiento fue efectuado con el fin de realizar recomendaciones en la siguiente campaña de perforación. Este cubo $3 \mathrm{D}$ ha permitido obtener una mejor idea de la zona de estudio y poder tomar decisiones en la perforación.

Mediante este estudio de IP se ha determinado la existencia de una anomalía de cargabilidad en profundidad que está asociada a una mineralización de sulfuros, destacando que la zona con resistividades bajas está relacionada a zonas de infiltración con fuerte fracturamiento y alto grado de alteración que origina una disminución en sus valores de resistividad, mientras que valores altos en resistividad corresponderían a rocas de mayor dureza.

Los resultados de este proceso muestran que las variables espectrales tales como la amplitud, fase y frecuencia ofrecen un valor adicional a los datos de cargabilidad. Las variaciones de las amplitudes generadas por la transformada nos muestran que los valores de amplitud definen muy claramente el límite de los dos bloques por donde pasa una falla de carácter regional. Además, se definen dentro del primer bloque tres pequeños bloques y que coinciden con dos fracturas transversales a la falla principal.
Los valores de fase dentro del cubo 3D, muestran cierta diferenciación y coinciden con la anomalía de la cargabilidad por lo que puede utilizarse esta variable para poder identificar zonas de mineralización de sulfuros.

El resultado en el procesado e interpretación del cubo 3D ha tenido como objetivo principal poder observar en un mismo dominio ya normalizado los cambios de resistividad, cargabilidad y las variables espectrales. Finalmente, seleccionar las zonas más favorables para el emplazamiento de sondeos y la identificación de zonas de alteración y zonas de fracturas y fallas.

Estos estudios mostraron resultados positivos, lo que permitió inferir la presencia de un cuerpo en profundidad con alto grado de cargabilidad y baja resistividad. Esta respuesta física es propia de los pórfidos profundos con alto grado de mineralización dentro de un cuerpo porfirítico, lo que hace de este proyecto un buen candidato para una campaña de perforación y logeo.

Con los datos registrados se ha generado hasta 8 cubos $3 \mathrm{D}$ con las siguientes variables que fueron obtenidas de las líneas registradas, utilizando las curvas de decaimiento.

- Cubo 3D con la variable Fase Normal (ver Figura 4)

- Cubo 3D con la variable Fase Logaritmo (ver Figura 5)

- Cubo 3D con la variable Amplitud Logaritmo (ver Figura 6)

- Cubo 3D con la variable Amplitud Natural (ver Figura 7)

- Cubo 3D con la variable Cargabilidad (ver Figura 8)

- Cubo 3D con la variable Resistividad Logaritmo (ver Figura 9)

- Cubo 3D con la variable Cargabilidad filtrada (ver Figura 10)

- Cubo 3D con la variable Tiempo (ver Figura 11)

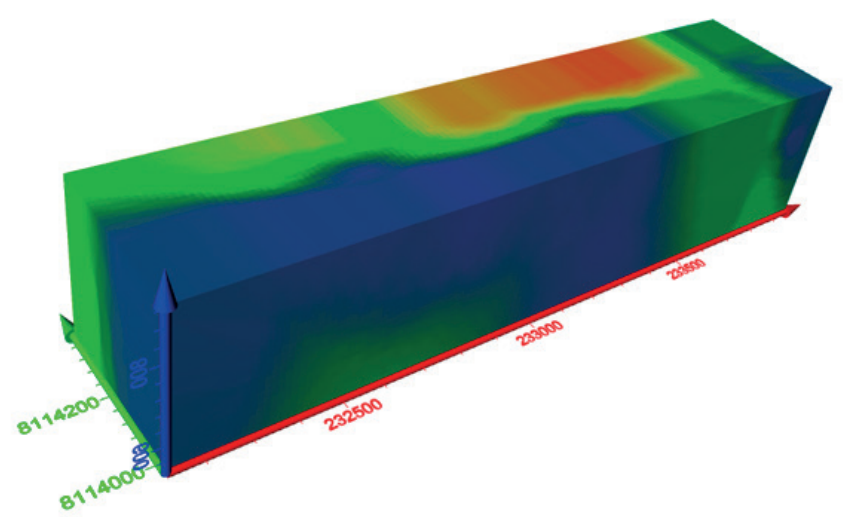

Figura 4. Cubo 3D con variable Fase Normal 


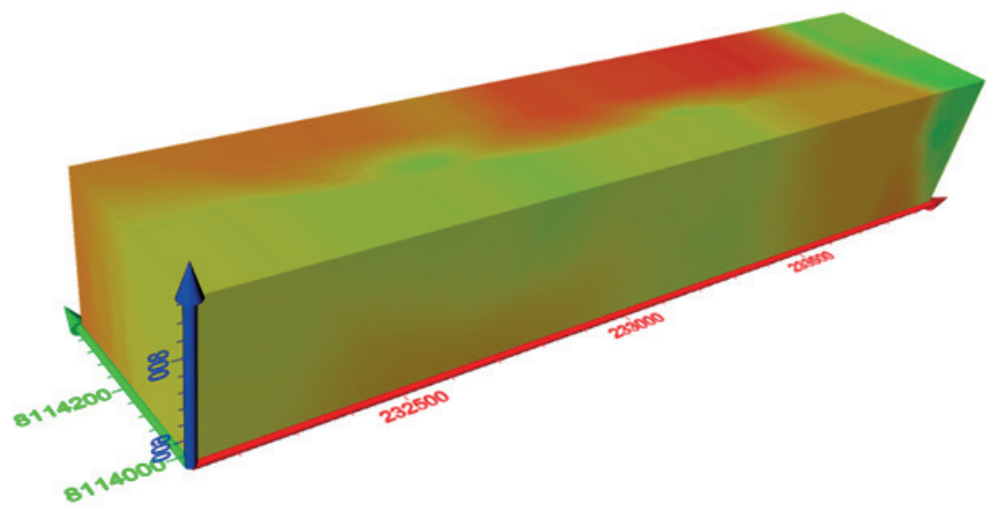

Figura 5. Cubo 3D con variable Fase Logaritmo

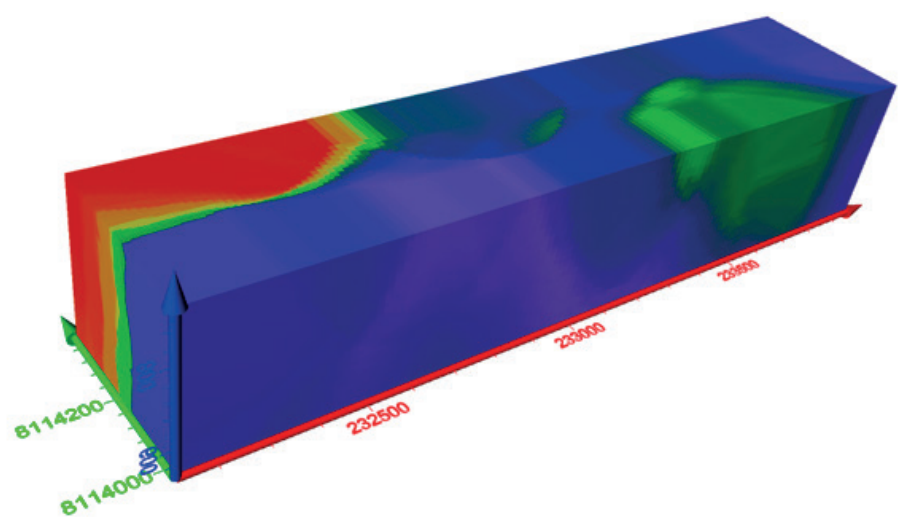

Figura 6. Cubo 3D con variable Amplitud

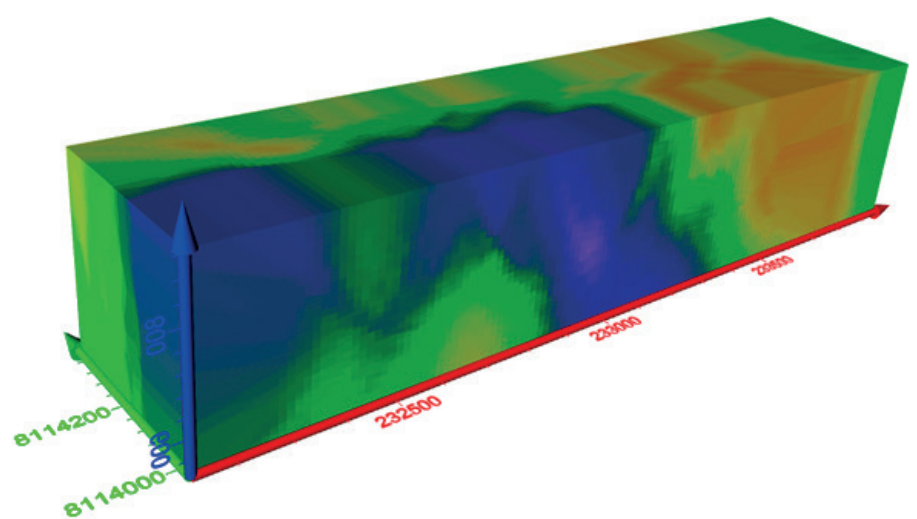

Figura 7. Cubo 3D con variable Amplitud Logaritmo

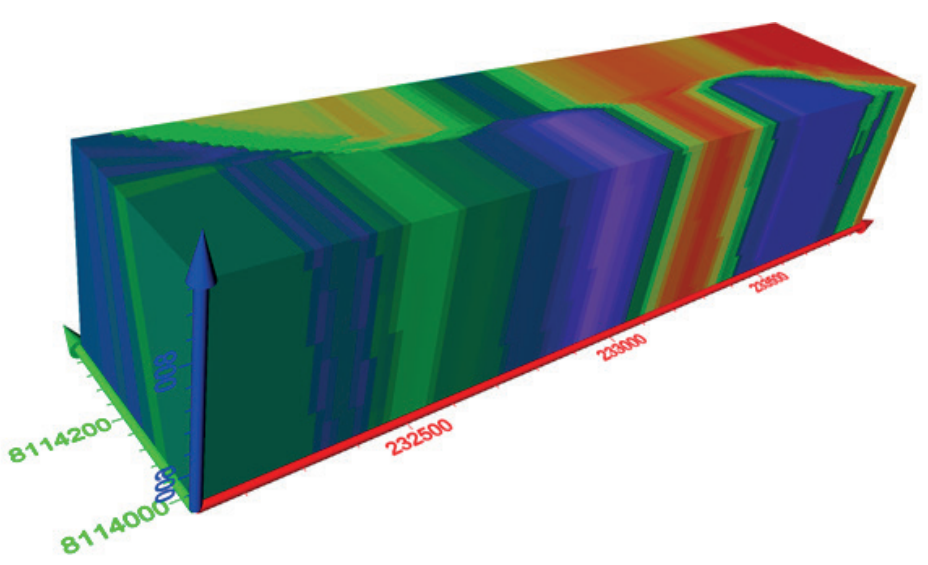

Figura 8. Cubo 3D con variable Cargabilidad 


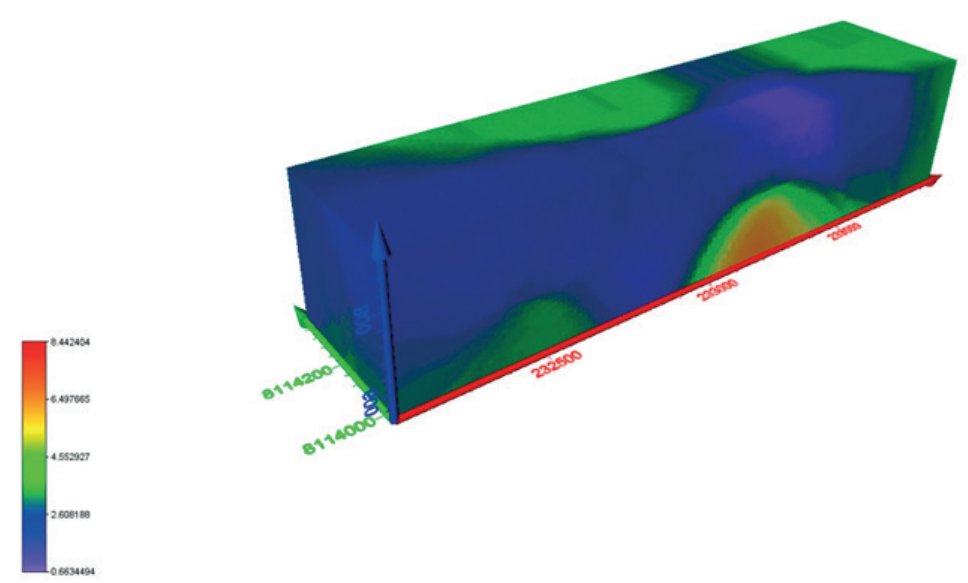

Figura 9. Cubo 3D con variable Fase Logaritmo

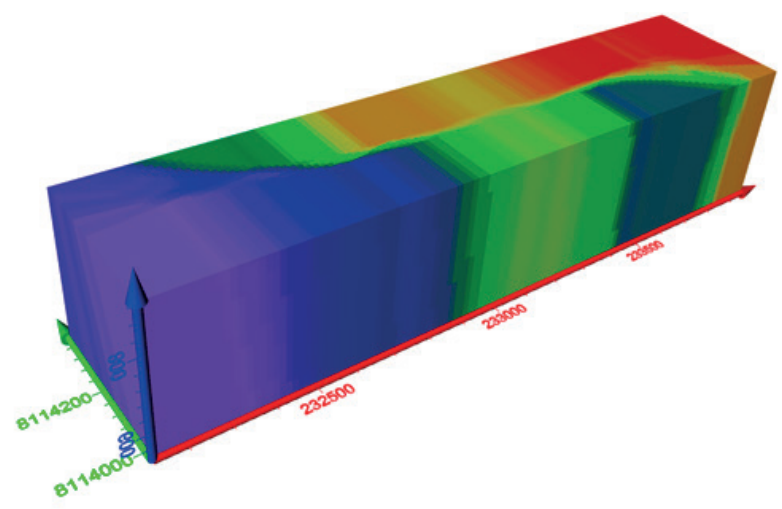

Figura 10. Cubo 3D con variable Amplitud

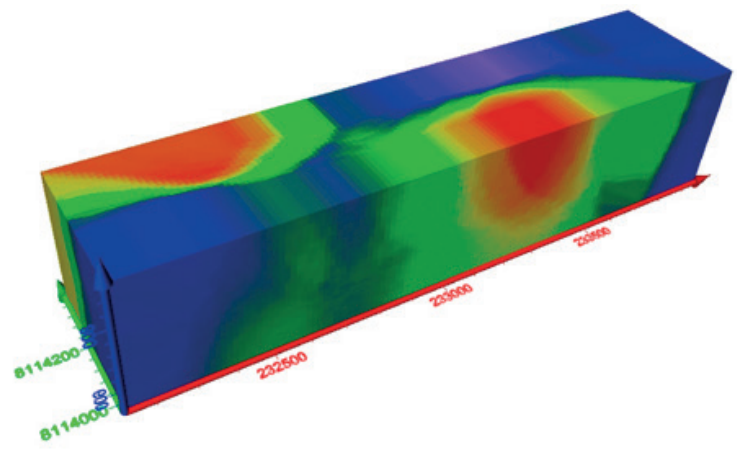

Figura 11. Cubo 3D con variable Amplitud Logaritmo

\section{DISCUSIÓN}

A partir de los cubos 3D obtenidos, aceptamos la hipótesis general y especifica que está orientada a encontrar una relación entre el valor de la cargabilidad y su valor en el dominio espectral, en este caso un valor de las variables de amplitud, fase y frecuencia. Mediante estos resultados se pudieron determinar e identificar zonas de mineralización en profundidad en la zona de estudio. De los resultados obtenidos, nuestro objetivo fue correlacionar la anomalía de cargabilidad encontrada en profundidad con alguna variable espectral. Esto se confirmó con la variable fase y esto ayudará en un futuro utilizar este método para identificar y caracterizar zonas exploratorias nuevas.

Estos resultados guardan relación con los que sostienen (Martin et al., 2020) donde recientes investigaciones han demostrado que mediciones en el dominio de la frecuencia (FDIP), tienen una dependencia de la frecuencia con la conductividad/resistividad compleja a través de mediciones recopiladas en el dominio del tiempo (TDIP). 
Al comparar la similitud entre las mediciones en el dominio de tiempo, frecuencia y fase como se usa en el método de polarización inducida para estudios de prospección geofísica, primero se deben definir los parámetros y establecer la base para la comparación. Las condiciones para la equivalencia matemática siguen luego en una secuencia natural. Las mediciones de laboratorio se realizan en una variedad de muestras de rocas para indicar el tipo de comparación y correlación que se espera de las mediciones de campo (ZONGE et al., 1972).

Los resultados indican que las mediciones de fase y dominio de frecuencia son equivalentes, pero se necesitan dos mediciones de dominio de frecuencia para producir la misma cantidad de información de polarización que una medición monofásica. Las mediciones en el dominio del tiempo y la frecuencia serán similares, pero nunca equivalentes debido a las diferencias básicas en sus respectivas definiciones de parámetros (ZONGE et al., 1972). Esto está acorde con los que se ha encontrado en este estudio.

\section{CONCLUSIONES}

- De los datos de cargabilidad registrados en el dominio del tiempo se ha extraídos datos que corresponden al dominio espectral y cuyas variables son la frecuencia angular, la amplitud y la fase

- Con estas nuevas variables hemos generado 3 cubos adicionales y esto ha hecho posible mostrar una mejor caracterización de la zona de estudio y estos resultados se ha utilizado en la parte de la interpretación geofísica.

- El uso de una mono frecuencia de 0.125 Hertz utilizada por el transmisor en la inyección de corriente a lo largo del registro de los datos de IP no ha limitado la obtención de nuevas frecuencias angulares que reflejan la respuesta del terreno en superficie como en profundidad. en superficie como en profundidad.

- Mediante este estudio fue posible confirmar la presencia de la falla regional Tambo- Locumba, la cual tiene una dirección Noroeste - Sur Este.

- Se obtuvo información sobre las propiedades físicas de la zona de estudio, en base a lo cual, se sustentó el desarrollo de un modelo geológicogeofísico que facilitará la interpretación de este estudio en la siguiente fase.

- Los valores de cargabilidad obtenidos en el cubo $3 \mathrm{D}$ varían entre 6 y 12 milivoltios/voltio y esto correspondería a un $0.4 \%$ de sulfuros, lo cual coincide con una zona en la que se presenta un dique en profundidad.

- Se realizo un buen control de calidad a los datos registrados y se aplicó un filtro digital a los datos para eliminar el ruido telúrico que se metió en la señal primaria y la distorsiono.
- Después de aplicar el filtro digital a las curvas de decaimiento, estas mostraron un comportamiento normal sin incluir valores negativos a estas curvas.

- Se obtuvo la función de transferencia utilizando los datos registrados y a través de la cual se ha podido extraer el contenido espectral a los datos de cargabilidad.

- Las anomalías de resistividad están controladas por fallas que presentan una pendiente de $60 \mathrm{y}$ 80 grados aproximadamente. Estos eventos son de moderados a débiles y estarían relacionados a procesos de fallamiento, así como con la presencia de cuerpos resistivos, fracturados y con control estructural, como se muestra en la sección de resistividad.

- Las anomalías de resistividad mostraron valores moderados a bajos, exponiendo mayores valores a mayores profundidades.

- En el cubo que se ha generado, el contenido de sulfuros totales es un factor de primer orden que explica las variaciones en la intensidad del efecto de la polarización inducida (cargabilidad eléctrica), obteniendo que la relación entre la concentración de sulfuros y su cargabilidad eléctrica presenta en general una proporcionalidad directa, pero no muestra una relación lineal. Por lo tanto, se puede establecer, que en este caso los valores de cargabilidad fueron de un valor moderado a bajo.

\section{AGRADECIMIENTOS}

Agradecimientos a la empresa SGA GEOFISICA por haber proporcionado los datos del estudio de polarización inducida. Asimismo, a todas aquellas personas que han apoyado en la revisión, evaluación y chequeo de este trabajo de investigación.

\section{REFERENCIAS}

Aguilef Carmona, S. G. (2012). Analisis geológico de estudios geofísicos de Polarización Inducida (IP) en sistemas IOCG y pórfido cuprifero del Norte de Chile. In Universidad de Chile. Universidad de Chile.

Bellido Bravo, E., \& Narvaez, S. (1960). Geología del cuadrángulo de Atico (Hoja 33-o)-[Boletín A 2]. Repositorio Institucional INGEMMET, 2. https://repositorio.ingemmet. gob.pe/handle/20.500.12544/32

Dentith, M. C., \& Mudge, S. (2015). Geophysics for the mineral exploration geoscientist. Choice Reviews Online, 52(06), 52-3156-52-3156. https://doi.org/10.5860/choice.186586

Martin, T., Günther, T., Orozco, A. F., \& Dahlin, T. (2020). Evaluation of spectral induced polarization field measurements in time and frequency domain. Journal of Applied Geophysics, 180. https://doi.org/10.1016/j. jappgeo.2020.104141 
Quinquis, A. (2013). Digital signal processing using MATLAB ${ }^{\circledR}$. In Digital Signal Processing Using $M A T L A B \circledR$ (3rd ed.). Global Engineering: https://doi. org/10.1002/9780470610992

Seigel, H., Nabighian, M., Parasnis, D. S., \& Vozoff, K. (2007) The early history of the induced polarization method Leading Edge (Tulsa, OK), 26(3), 312-321. https://doi. org/10.1190/1.2715054

Sheriff, R. E. (2002). Encyclopedic Dictionary of Applied Geophysics. In Encyclopedic Dictionary of Applied Geophysics. https://doi.org/10.1190/1.9781560802969

Sogade, J. A., Scira-Scappuzzo, F., Vichabian, Y., Shi, W., Rodi, W., Lesmes, D. P., \& Morgan, F. D. (2006). Inducedpolarization detection and mapping of contaminant plumes. Geophysics, 71(3), 71. https://doi.org/10.1190/1.2196873
Telford, W., \& Geldart, L., \& Sheriff, R. (1931). Applied geophysics. In Nature (Vol. 127, Issue 3212, pp. 783-785). https://doi.org/10.1038/127783a0

ZONGE, K. L., SAUCK, W. A., \& SUMNER, J. S. (1972). COMPARISON OF TIME, FREQUENCY, AND PHASE MEASUREMENTS IN INDUCED POLARIZATION. Geophysical Prospecting, 20(3), 626-648. https://doi. org/10.1111/j.1365-2478.1972.tb00658.x 